\title{
Communication Et COVID-19 Au Sénégal. Approche Pragmatique Et Énonciative De Slogans Et Concepts De Lutte Contre La Pandémie
} Daouda NGOM ${ }^{1 *}$

\author{
${ }^{1}$ Docteur en Sciences du langage. Spécialité: Analyse du discours.Université Cheikh Anta Diop de Dakar, Sénégal
}

DOI: $10.36348 /$ sijll.2021.v04i03.003 | | Received: 05.03.2021 | Accepted: 23.03.2021 | Published: 30.03 .2021

*Corresponding author: Daouda NGOM

\section{Abstract}

Senegal has been plunged in resolving the health crisis of the COVID-19 pandemic since its appearance in March 2019 on Senegalese territory. All useful means such as communication are pointed up to cut the line of contagion of the virus. In this communication fight against the COVID-19 pandemic, we noted the presence and use of concepts, slogans and / or "sloganized" expressions of which understanding of their communicative values requires an operation of interpretation and decoding. The perception of the informative content of such speech acts is based on their context of use by the communicating subjects. In the pragmatic theory of language where the communicational approach is based on fundamental notions: the implied and the presupposed, the interpretation of the communicative value is done through a set of values, common to the communicating subjects, which guarantees the intercomprehension of messages. This article aims, from a pragmatic and enunciative angle, to enumerate the slogans and concepts created in the management of the pandemic and then to identify their new semantic particularities, and to decipher their preventive implicit content in the communication of the fight against COVID-19. Particularities that are similar to calls for awareness on the existence and seriousness of the disease.

Keywords: Communication, COVID-19, pandemic, slogan, concept, pragmatics, enunciation.

Copyright $\odot$ 2021 The Author(s): This is an open-access article distributed under the terms of the Creative Commons Attribution 4.0 International License (CC BY-NC 4.0) which permits unrestricted use, distribution, and reproduction in any medium for non-commercial use provided the original author and source are credited.

\section{INTRODUCTION}

Depuis le mois de décembre 2019, l'univers, dans sa globalité, subit violemment sur tous les plans les fouets d'une des pandémies jamais connues de tout le monde : le COVID-19. L'apparition de la maladie à coronavirus à Wuham en Chine a fini par circonscrire le monde entier, créant des bouleversements et des mutations forcées dans tous les domaines d'activité de l'homme. Le monde est ainsi appelé à faire face à un adversaire aussi véloce qu'inarrêtable sur le plan de la propagation. C'est le qui-vive dans les tous pays, des grandes puissances aux pays sous-développés en passant par ceux en voie de développement. Chaque pays se barricade, gère sa communication pour trouver une issue de sortie.Des communications conflictuelles naissent donnant lieu à des mésententes diplômatiques dont l'origine relève de la crise sanitaire du COVID 19. On se rappelle la guerre communicationnelle, par presse interposée, entre le Président des Etats Unis d'Amérique et son homologue chinois sur la question de la paternité du virus avec la formule laconique de Donald Trump «the chinese virus » (le virus chinois). Cette formule, qui finit par prendre les allures d'un slogan, est amplifiée par les médias nationaux et internationaux sur la présentation de la pandémie, consiste à accuser la Chine d'être propriétaire du virus. Elle insinue, par ailleurs, la responsabilité et la négligence des autorités chinoises sur la gestion de la propagation du virus dans le monde.

$\mathrm{Vu}$ la vitesse exponentielle de la propagation de la pandémie, des discours à la nation, assortis de slogans, de divers présidents s'intensifient. Le « nous sommes en guerre » déclaré par le Président de la France, Emmanuel Macron en est une belle illustration de l'engagement et la détermination des autorités politiques dans la gestion de la crise. Ce slogan appelle le peuple français à comprendre la dangerosité du virus et la gravité de la situation.

Au Sénégal, le Président Macky Sall emboite le pas à son homologue français en reprenant le slogan dans son premier discours à la nation sur la question de l'actuelle crise sanitaire en l'accordant le même contenu communicatif : mesurer l'ampleur de la gravité de la situation causée par le virus. On assiste ici une opération de mise en garde, de prévention de la part des 
Daouda NGOM., Sch Int J Linguist Lit, Mar, 2021; 4(3): 80-87

sujets communicants à travers l'usage de ce slogan. Ils informent leurs peuples respectifs, autrement dit, ils communiquent avec leurs peuples sur la question. Communiquer c'est échanger des informations, des messages, etc. Ces messages sont particuliers par la notion et l'évaluation $\mathrm{du}$ risque sanitaire. La communication doit, dans ce cas, être véridique et utile pour les citoyens: «elle doit être continue, transparente, indépendante, crédible et utilisable par le citoyen $\gg\left[{ }^{1}\right]$.

C'est pour dire qu'une communication en temps de crise doit être bien étudiée et programmée par rapport aux enjeux de la situation : «toute stratégie visant une planification communicationnelle doit prendre en considération les règles inhérentes à une crise sanitaire. $\gg\left[{ }^{2}\right]$. Cet état de fait prouve clairement combien la communication est déterminante dans la gestion d'une crise, quelle qu'en soit; c'est ce que précise Thierry Libaert en ces termes «La crise est multiforme. Elle concerne les domaines technologiques, sociaux, réglementaires, financiers, commerciaux ou écologiques, mais s'appréhende d'abord sous l'angle de la communication. Celle-ci apparaît omniprésente et les médias, qu'ils soient traditionnels ou on line construisent autant l'événement qu'ils l'amplifient. Au cœur du dispositif de gestion de crise, la communication est l'élément déterminant qui permet, selon sa plus ou moins bonne maîtrise, de surmonter la crise. » $(2015, \mathrm{p} 7)$.

Dans la présente crise sanitaire, nous avons remarqué que certaines expressions «sloganisées » s'inscrivent dans la logique du respect des règles inhérente à la pandémie. Des énoncés tels « restez chez vous ! le corona il est dehors », « lavez vous les mains pendant chaque trente minutes », «portez un masque », etc. entrent dans le cadre de la présentation de la nature de la maladie (contagieuse, transmissible...), et décrivent les formes de moyens de lutte qui s'imposent à tout le monde. De tels slogans, sous le mode injonctif, constituent le marquage du début des hypothèses évoquées sur les canaux par lesquels le virus se répand. C'est la première phase de la pandémie, de ce fait, la communication est centrée sur les caractéristiques, la description et les voies de propagation de la pandémie.

Certains slogans du type «Soyez prudent! le COVID 19 est toujours là » sous-entendent un certain ralentissement de la crise. Ils font état d'une certaine maîtrise ou d'une main mise sur la gestion de la crise sanitaire. D'autres tels que "protégez vous pour nous protéger!», annoncent une nouvelle tendance augmentative de la pandémie. Ceux du type, « ensemble pour vaincre le COVID 19 » appellent à la

\footnotetext{
${ }^{1}$ Eddine K. J et Cie, Crise sanitaire et stratégie de communication, septembre 2020, p 2

${ }^{2}$ Eddine K J et Cie, ibid.
}

mobilisation citoyenne autour de la question. D'autres énoncés à l'image de "Portez un masque, le corona tue ! » mettent en garde ou tout simplement alertent sur les conséquences néfastes de la maladie tout en engageant la responsabilité de chaque citoyen.

Nous analysons dans cet article toutes ces valeurs communicatives, du point de vue pragmatique et énonciatif, qui découlent de l'utilisation des slogans dans la communication sur la lutte contre la pandémie du COVID 19. Les exemples de slogans cités haut démontrent à foison la situation sanitaire critique dans laquelle se baignent plusieurs pays du monde tels que le Sénégal. Des slogans dont les valeurs communicatives implicites ne peuvent être décryptées qu'en tenant en considérant le contexte actuel et la phase d'évolution de la crise dans laquelle ils sont employés.

Nous essayerons dans cette analyse non seulement de nous livrer, au-delà de l'aspect typologique des slogans, à un exercice d'interprétation pragmatique et sémantique mais aussi à une analyse énonciative en vue de décrypter la valeur communicative de ces slogans. Cette gymnastique interprétative et cette démarche énonciative, combinées, nous permettront de ressortir le sens dénotatif de ces unités lexicales, c'est-à-dire les nouvelles attributions sémantiques et communicationnelles (les significations nouvelles) qu'ils comportent. L'analyse se doit de ressortir la valeur pragmatique (et sémantique) et la visée discursive de telles expressions en termes de persuasion, de sensibilisation, de prise de conscience.Et l'analyse énonciative des éléments de langage qui constituent les slogans permettra d'arriver à une meilleure compréhension des desdits slogans.

Ces approches combinées nous permettrons de nous livrer non seulement à une étude thématique et énonciative des slogans mais aussi à une interprétation pragmatique pour déterminer la fonction communicative et la valeur pragmatique des actes de langage analysés en contexte de communication. Selon Michel Hupet (1993), cette approche basée sur ce qu'on appelle «signification de phrase » (sentence meaning) dont s'occupe la sémantique, et «sens de l'énoncé » (utterance meaning) dont s'occupe la pragmatique, permettra de partir du sens premier à la signification contextuelle des concepts et énoncés décryptés dans ce travail. Les «calculs interprétatifs » de ces branches de la linguistique, permettront également de ressortir l'implicitation (le sous-entendu et le présupposé) que comportent ces actes de langage, autrement dit, les effets des actes illocutoires (l'intentent du locuteur) et perlocutoire (effets psychologiques) de ces énoncés chez le destinataire (la population).

\section{Démarche Méthodologique}

Le slogan comme acte de langage a longtemps retenu l'attention des chercheurs et spécialistes aussi bien en communication politique qu'en communication 
Daouda NGOM., Sch Int J Linguist Lit, Mar, 2021; 4(3): 80-87

syndicale, bref sur toutes les formes de communication. Défini comme «une formule concise et frappante, utilisée par la publicité, la propagande politique, etc. $»\left[{ }^{3}\right]$, le slogan, de par sa force occulte et de frappe, joue une multitude de fonctions discursives. De l'attention à la persuasion de l'auditeur, de l'auditoire, de l'interlocuteur ou du récepteur, ces formules simples (faciles à répéter et à mémoriser) constituent un recours déterminant dans l'exercice communicationnel du sujet communicant. Leur spécificité liée à leur style simple, fait que leur usage dans la communication sociopolitique, professionnelle, institutionnelle, syndicale, entre autres, fascine les experts en analyse de discours et en communication.

Les recherches sur la communication de façon générale sont en plein boum depuis la fin des années soixante, avec la poussée fulgurante des sciences du langage et de la communication, marquée par des courants comme l'ethnographie de la communication, l'ethnométhodologie de la communication, la pragmatique communicationnelle, l'analyse du discours, entre autres. Les spécialistes tels que les linguistes analystes du discours, les sociolinguistes, en ont fait leur domaine de prédilection pour décrypter les éléments constitutifs de la communication. La communication dans les domaines sociopolitiques ou en temps de crise a intéressé les chercheurs de par leurs enjeux et autres particularités relatifs aux circonstances de communication des différents acteurs. Les enjeux sont énormes et leurs particularités reposent sur les discours conflictuels des protagonistes et cela dans beaucoup de secteurs d'activité. Sur le plan politique, Patrick Charaudeau précise que les «discours politiques entrent en collision une vérité des apparences mise en scène par le discours et une vérité des actions mise en œuvre par des décisions $»\left[{ }^{4}\right]$.

Et Daouda Ngom d'ajouter que « la finalité principale de cette communication c'est de se faire comprendre, de persuader l'opinion publique $»\left[{ }^{5}\right]$. Dans l'espace éducatif, surtout lorsqu' il y a crise, " c'est sur le terrain de la communication que se nouent localement une grande partie des rapports de forces entre salariés et employeurs » $\left[^{6}\right]$ ou bien entre étudiants

\footnotetext{
${ }^{3}$ Le Grand Robert de la langue française

4 CHARAUDEAU P., Le discours politique. Les masques du pouvoir. Paris : Vuibert, 2005, p : 83.

${ }^{5}$ NGOM D, «Langues nationales et communication politique au Sénégal, Essai typologique des stratégies de persuasion à travers quelques dénominations wolof données aux coalitions politiques des élections locales du 29 Juin 2014 : le cas du département de Dakar » in Langues et Littératures, Revue du Groupe d'études linguistiques et littéraires, $\mathrm{n}^{\circ} 2$, Janvier 2017, p : 45-65, UGB, Sénégal.

${ }^{6}$ Stéphane Olivesi : La communication syndicale, Paris, PUR, 2013, p : 12.
}

et Etat. En temps de guerre ou de crise sanitaire, la communication constitue la pièce maîtresse dans le processus de la résolution des conflits ou de la lutte contre les pandémies. Dans de telles situations, des prises de positions politiques et des prises de décisions s'imposent à tous les niveaux de la communauté, du sommet de l'Etat à la cellule familiale. L'expérience des crises traversées montre que la communication reste un maillon crucial dans la gestion desdites crises. Elle représente une chose non négligeable dans des situations sanitaires à l'image du COVID 19. C'est ce que Kohen Jamal Eddine et Cie expliquent à propos de la pandémie COVID-19 en ces termes : « elle constitue une situation inédite de crise sanitaire en santé publique nécessitant une gestion appropriée de l'information des citoyens qui est une exigence éthique et démocratique d'une part, et une condition d'efficacité de gestion des crises sanitaires. » $(\mathrm{p} 2)\left[^{7}\right]$.

Dans la gestion d'une crise sanitaire, toutes les stratégies de planification communicationnelle prennent forme en fonction des normes relatives à une crise sanitaire. Le recours aux moyens discursifs que la langue nous offre devient inévitable. Les acteurs de la gestion de la pandémie puisent dans leur plus profond répertoire pour trouver les mots, termes et expressions pouvant efficacement participer à la sensibilisation pour la lutte contre la propagation du COVID-19. L'usage de slogans semble approprié à cet effet. Les slogans s'érigent en vedette et de toutes sortes partout : «nous sommes en guerre »du Président français Emmanuel Macron, repris par beaucoup de ses homologues africains dans leurs discours à la nation durant la pandémie.

Les slogans de lutte contre le COVID 19 sont des actes de langage à fonction informative et préventive. Pour son analyse interprétative formelle, nous adopterons le modèle d'analyse que propose le spécialiste de la télécommunication, C. F Shannon, pionnier de la théorie de la communication. Un modèle construit à partir de cinq paramètres présents dans un exercice de communication: l'émetteur du message (politiques, syndicaliste, apprenant), le récepteur (le destinataire, ici, Etat, population), le canal (le moyen pour faire passer le message : ici la voix ou l'écrit), le message (le contenu informationnel, il s'agit ici de détournement de sens, de création lexicale), le code (ici la langue française et wolof). L'identification de la création lexicale, autrement dit, que veut dire l'énoncé nouvellement créé, et la découverte de la signification énonciative, c'est-à-dire que veut dire celui qui produit l'énoncé seront les étapes du processus d'interprétation que nous comptons faire pour les slogans de notre corpus.

\footnotetext{
${ }^{7}$ Eddine K. J et Cie, Crise sanitaire et stratégie de communication, septembre 2020, p 2.
} 
Daouda NGOM., Sch Int J Linguist Lit, Mar, 2021; 4(3): 80-87

Nous ne pouvons pas nous empêcher d'épouser l'approche de la pragmatique sur le dire en contexte social pour dégager l'aspect implicite des expressions analysées dans le travail. La pragmatique met en connexion les actes de langage à l'intention communicative du sujet-communicant. Ici, les actes de langage englobent l'intégralité de la production discursive. Pour Momar Cissé, la conception de cette théorie «annule la représentation de l'échange communicatif sous forme d'un système d'équipollence encodage / décodage. En effet, elle situe la pratique discursive dans la mouvance de la subjectivité dont elle prend en charge les différentes manifestations.Les incidences d'une telle approche sur la conception de la communication sont évidentes $\gg\left[{ }^{8}\right]$.

Il n'est pas ici question de faire une analyse de ces incidences; nous essayons seulement de montrer l'intention du communicant à travers la création lexicale de ces slogans et de ressortir les composantes sémantiques et les divers phénomènes de subjectivité dépendant de l'actualisation spatio-temporelle des desdits slogans.

La problématique de l'usage des slogans comme procédés de lutte contre la propagation des épidémies comme le COVID 19 et surtout comme stratégies discursives de sensibilisation dans la gestion sociopolitique de telles pandémies n'a pas été prise en compte par les travaux antécédents, du moins au Sénégal. L'article de Momar Cissé, cité haut, interprète le sens du slogan politique dans ses relations avec les conditions de production et le système de pensée de l'auteur mais également du public récepteur. Dans son article, il n'est question pas d'interpréter l'apport et la valeur communicative, et la création lexicale du slogan, comme nous le proposons ici. Toutefois, il participe à la compréhension générale des phénomènes de subjectivité et de l'élaboration des hypothèses de sens du slogan. Ceci étant, nous estimons que des études doivent, aujourd'hui, être orientées vers d'autres aspects non encore explorés de la communication en temps de crise sanitaire au Sénégal.Une telle orientation croiserait de fort belle manière les nouvelles tendances de la linguistique telle que l'analyse du discours politique ou syndical, la pragmatique communicationnelle, entre autres.

Ce travail qui porte sur la valeur ajoutée, le renfort ou l'impact des slogans dans la communication sociopolitique en situation actuelle de crise sanitaire, pose la problématique de l'interprétation du dire dans des situations de crises naturelles entre les décideurs étatiques et la population. Se pose également le

${ }^{8}$ Cisse M, (2007). «Campagne électorale sénégalaise de Février 2007. De 1 usage des slogans wolof comme mode de contestation de la langue-code.» in SudLangues $\mathrm{N}^{\circ} 7$, p 73-95. problème de l'analyse formelle des slogans. La subtilité langagière (changement, glissement de sens, substitutions lexicales, etc.) notée dans le discours de ces acteurs s'inscrit dans une logique de persuasion, de sensibilisation, de prévention et pourrait poser un problème de compréhension spontanée d'une tierce personne. L'article identifie et analyse tous ces aspects d'une communication de crise en contexte sénégalais sous l'angle pragmatique et énonciatif. L'analyse pragmatique félicitera la compréhension et le décryptage des informations sous-entendues ou présupposées et la force illocutoire dont elles génèrent. L'énonciation étant l'action d'énoncé par la parole ou par écrit en situation de communication, l'approche énonciative sera d'une grande importante dans la prise en compte du contexte d'énonciation pour l'interprétation des concepts et slogans étudiés dans ce texte.

La collecte des données exploitées dans le travail a été effectuée par nous-mêmes à travers des spots publicitaires, des bandes d'annonces, des banderoles, des communiqués quotidiens du ministère de la santé et de l'action sociale, des différents discours à la nation du Président de la république et des enseignes des campagnes de sensibilisation dans le cadre de la lutte et de la résilience contre la pandémie au Sénégal. Certains slogans sont recueillis des débats politiques télévisés. Il nous arrive, en toute amitié, de discuter des questions sur tel ou tel concept pour avoir beaucoup plus de certitude sur l'emploi et la signification effective des concepts «sloganisés » et des slogans en situation de crise.

Certains des slogans et concepts recueillis dans ce travail présentent une particularité communicationnelle et d'autres ont un pouvoir extrêmement efficace de dissuasion et de mise en garde dont le décryptage nécessite une connaissance des composantes de l'implicite du discours des acteurs en de pareilles situations de communication. Dans ce travail, l'approche typologico-interprétative que nous employons pour les productions de slogans de dissuasion, de mise en garde, entre autres, se fonde sur leurs circonstances générales de production en rapport avec l'évolution et la gestion de la pandémie au Sénégal.

\section{Slogans, Comme Procédés De Lutte Contre La Pandémie}

Décidément inscrite dans une perspective à la fois interprétative et pragmatique, cette rubrique met en exergue la typologie des slogans et leurs fonctionnalités dans la communication «coronale » au Sénégal. Il sera ici question de ressortir le rôle particulier de ces unités lexicales dans la communication sur la présentation, les caractéristiques, les conséquences de la pandémie au sein de la société. 
Daouda NGOM., Sch Int J Linguist Lit, Mar, 2021; 4(3): 80-87

2.1: Enoncés «sloganisés » et phases de production dans le déroulement de la lutte contre le COVID-19

\subsection{1: Cadrage de la situation et description du COVID 19}

La peur est un sentiment tout comme la joie, elle découle de la vue ou de l'attente du danger. L'annonce du premier cas testé positif au coronavirus, le 2 mars 2020, au Sénégal, avait suscité des frémissements au sein de toute la nation. La peur du danger imminent, l'anxiété, et l'angoisse amplifiées par les médias et les réseaux ont fini par créer la psychose totale dans le pays. Un mode de communication planifié s'impose aux autorités afin de rassurer la population. Malheureusement, dans la phase 1 de la pandémie, le slogan injonctif Restez chez vous a plus apeurer que rassurer au Sénégal. Son usage semblait inapproprié en début de crise dans un pays où le secteur informel polarise plus de la moitié des travailleurs. La visée communicative de cette injonction qui consistait d'alerter les citoyens sur la dangerosité et la rapidité à se répandre du virus a été spontanément submergée par la peur des uns et des autres de perdre leur emploi. Elle était conçue plus contraignante que consolante. Il urge donc d'établir un lien de confiance établi entre l'autorité tutelle et le peuple. Le ministère de la santé et de l'action sociale initie une rencontre quotidienne face à la presse, dénommée «le point du jour» sur la situation du jour afin relayer les bonnes informations et contrecarrer la fulgurante vague des «fake news » (fausses informations) des réseaux sociaux.

L'Etat déroule ainsi un plan de riposte et mobilise aussi bien des moyens financiers que communicatifs. Les moyens linguistiques sont mis en branle. Convaincu que l'analphabétisation atteint toujours des proportions élevées malgré les efforts consentis, en plus du français, langue de l'enseignement et de l'administration, l'Etat implique toutes les langues nationales pour atteindre toutes les cibles : la population entière. C'est le début des campagnes de sensibilisation sur les mesures édictées par les autoritaires sanitaires et paramédicales sénégalais. Et cela exige un casting pour définir le communicant et les informations à communiquer aux destinataires, exemple le peuple. De là relève l'importance d'un style communicatif «sloganisé » si l'on comprend que dans la communication sur la gestion d'une telle crise, les messages, ordres et suggestions doivent être concis, clairs, courts, cohérents «et bien expliqués pour une bonne assimilation par les citoyens surtout ceux concernant les mesures impactant la vie socioéconomique et les libertés ». Toute sorte d'ambigüité doit être évitée.

Le style de slogans semble plus approprié, vu leur simplicité langagière accessible et à la portée de tous. Ils sont faciles à mémoriser, à répéter et à assimiler. Les acteurs pour la sensibilisation pour lutter contre la propagation du virus tels que les musiciens (rappeurs et mbalaxmen ${ }^{9}$ ) impliqués lors des caravanes de sensibilisation, l'ont bien compris. Les gestes barrières, reprises en langue française ou traduites en langues nationales comme le wolof (langue véhiculaire, majoritairement parlée au Sénégal), font l'objet de chansons, d'affiche et d'enseignes sur fond de slogans. Des slogans visant à décrire la maladie et ses formes de contagion naissent. On note certains comme takkal sa mask ! koronaa defa sew (Porte ton masque, le corona est mince ». Ce slogan renvoie au caractère d'invisibilité du virus et surtout il insinue ses possibilités à infecter facilement les gens. L'énoncé wolof defa sew traduit une sorte de mépris que l'on a contre le virus. Le verbe sew en wolof signifierait mince, petit, (physiquement ou dans le comportement)

Takkal sa mask est un ordre sous le mode du tutoiement, l'énonciateur s'adresse explicitement et de façon directe au citoyen pris individuellement. Une manière de pousser chacun à se sentir concerné par le port de masque. L'énoncé bul joxe loxo, koronaa defay wale (Ne serre pas la main (à l'autre)! le corona est contagieux), joue le même rôle, il est à titre informatif et descriptif sur les voies de transmission du virus.

\subsection{2: Slogans inclusifs ou associatifs}

Plus que le nombre de cas testés positifs au coronavirus augmente plus les autorités évaluent le processus communicationnel pour apporter des réajustements et réadaptations afin de dominer la situation. L'implication totale de toutes les composantes de la société s'avère indispensable. Le discours doit désormais être orienté vers l'inclusion de tous étant donné que «la communication dans la gestion d'une crise sanitaire exige une approche transparente, réaliste, inclusive qui garantit l'acceptation (passivité) et l'adhésion des destinataires aux contraintes imposées par le contexte / activisme » (Eddine et Cie, p 11). Du coup des slogans tels que And faxas koronaa (Ensemble pour .....), And xeex koronaa (Ensemble pour vaincre le corona), Opération daan COVID (Opération terrasser COVID) entrent en vigueur. On cherche à impliquer de façon directe et active les citoyens. On leur tend la main dans la lutte contre le virus. Une telle démarche exprime une sorte de considération des autorités envers les destinataires: les populations. Se sentant considérées, ces dernières pourraient mieux s'impliquer farouchement et surtout de façon désintéressée dans la gestion de la crise. Une telle formule de communication établit un lien de confiance entre les acteurs. L'absence de confiance pourrait biaiser l'assimilation des messages. Eddine et Cie posent la problématique de la confiance en situation de crise sanitaire «dans une situation de risque sanitaire, la confiance est un état cognitif complexe caractérisé par un mélange de

\footnotetext{
9 Musiciens qui chantent le «mbalax » qui est une musique traditionnelle sénégalaise.
} 
Daouda NGOM., Sch Int J Linguist Lit, Mar, 2021; 4(3): 80-87

rationalité, de sentiments, d'engagement au sujet d'une relation avec le communicateur (l'institution). » (p 13).

De telles unités lexicales visent la sensibilité des citoyens sous une apparence associative et inclusive. Elles sont faites sous la forme d'invite et de suggestion de s'unir pour une meilleure gestion de la propagation du virus. Dans ce type d'appellations, on remarque toujours la présence d'un verbe ou de terme à valeur associative, inclusive et coopérative. C'est le rôle que joue le membre de phrase en gras dans : Ensemble pour vaincre la COVID 19, (slogan de la Banque de l'Habitat du Sénégal). L'adverbe ensemble qui veut dire l'un avec l'autre, les uns avec les autres, constitue ici un appel à l'unité pour faire face à la pandémie. Ce qui sous-entend que la séparation des efforts des uns et des autres n'est pas l'idéal dans ce contexte de pandémie. Booloo xeex korona et And daan korona, deux slogans populaires qui insinuent la mutualisation des efforts pour lutter contre la pandémie. Les verbes booloo qui signifie en wolof s'unir, s'accorder ou se mettre d'accord en vue d'accomplir une tâche et and qui traduit, entre autres, le fait d'être ensemble, d'aller ensemble, représentent une invite à la collaboration pour plus de force et de résilience dans la lutte. Cette volonté ou souhait d'être plus forts et plus résilients, se justifient par l'emploi des verbes xeex et daan dans les énoncés Booloo xeex korona et And daan korona qui veulent dire respectivement s'unir pour combattre le corona ; se mettre ensemble pour vaincre le corona. Le recours aux verbes xeex et daan démontre clairement qu'il s'agit d'un adversaire à abattre. Ici, Korona (écriture wolof) est l'adversaire à combattre, à vaincre dans un élan de solidarité collective.

La composition de ces énoncés verbaux booloo xeex et and daan comporte une force de frappe extraordinaire, et psychologiquement agit sur la population. Ce genre de formules présuppose une nécessité de collaborer pour une gestion collective et une mobilisation de toutes forces de la nation, partant de la cellule familiale au sommet de l'Etat dans l'optique de circonscrire la propagation du virus et de l'éliminer tout bonnement. L'usage de tels slogans suggère une union nationale autour de l'urgence sanitaire: la pandémie. On assiste ici à des choix lexicaux très forts du point de vue sémantique et surtout communicationnel, qui mettent en exergue l'urgence sanitaire du moment.

En communication de crise sanitaire, on met souvent l'accent sur la maladie, plus précisément sur les symptômes, la transmission, les moyens de protection, les personnes les plus vulnérables et enfin sur les voies de sortie de crise. On appelle le peuple à la prudence. C'est ce qui justifie l'emploi d'unités lexicales à valeur associative, une manière de dire à la population que c'est une affaire collective et que tout le monde doit s'y impliquer. Cette stratégie communicative correspond à un rappel destiné à la population pour attirer son attention sur la gravité de la situation. Les verbes Booloo et and jouent cette fonction associative. Ils s'opposent aux verbes à fonction dissociative ou exclusive tels que xaajoo (se séparer, se diviser), tasaaroo (s'éparpiller, se désunir) dont l'usage semble inapproprié dans la formation de slogans de lutte contre la pandémie.

\subsection{3: Appels à la prudence et à la responsabilité, sous forme de mises en garde}

Il est important de rappeler que la typologie des slogans ou énoncés sloganisés s'opère suivant l'évolution de la pandémie, autrement dit, selon la baisse ou l'augmentation des cas de contamination. Au Sénégal, le président de la République Macky Sall s'est adressé à la nation à plusieurs reprises dans le cadre de la gestion problématique voire contraignante de la pandémie et surtout pour exposer aux populations des difficultés économiques engendrée par la pandémie. Il est arrivé un moment où l'économie du pays était à genou, des pertes d'emploi notées ça et là, mettant l'Etat du Sénégal dans une situation financière intenable. Face à cette situation de crise économique causée par la crise sanitaire de la pandémie, le président a fait une adresse à la nation consistant à alléger les mesures de restrictions prises dans le cadre de la lutte contre la propagation du virus et a lâché la fameuse phrase «nous devons apprendre à vivre avec le virus ». C'est le début d'une nouvelle tournure dans la vie des sénégalais en temps de pandémie. L'assertion a fini par prendre les allures d'un slogan populaire car répétée ou reprise par les sénégalais comme justificatif de leurs actes et attitudes tendant à bafouer les mesures barrières. Cette «bombe » communicationnelle du président Sall est la résultante d'un relâchement inédit de la population par rapport à la prudence préconisée et les mesures édictées par les services sanitaires du ministère de la santé et de l'action sociale. Le segment vivre avec le virus sert dés lors de justificatif. L'emploi de cet énoncé présuppose, pour certains, comme un aveu d'échec du plan de riposte mis en branle par le gouvernement pour endiguer la propagation du virus. Le prononcer pour se dédouaner d'un manque de respect des mesures barrières, c'est comme imputer la responsabilité au président Sall, qui a demandé au peuple d' «apprendre à vivre avec le virus ». C'est slogan justifiant le relâchement du peuple relatif au respect des mesures barrières.

Malgré cette mauvaise interprétation du message à la nation du président Macky Sall, s'en suivi une tendance baissière de cas positifs de la première vague de contamination de la COVID 19, qui a également favorisé un laisser-aller, une négligence, une décontraction des masses par rapport au respect des mesures barrières : port correct $\mathrm{du}$ masque, distanciation sociale ou physique, lavage fréquent des mains, entre autres. Mais ce fut éphémère la tendance baissière. Une deuxième vague de contamination plus sévère frappe le Sénégal, suscitant psychose, angoisse, 
Daouda NGOM., Sch Int J Linguist Lit, Mar, 2021; 4(3): 80-87

et peur. La variante anglaise du virus en est l'origine, d'où la prise de nouvelles mesures de restriction de nouveau par le président de la République. Une nouvelle approche de communication se met en place et des slogans et énoncés faisant office d'alerte ou de mise en garde entrent en vigueur. C'est la stratégie communicative d'appel à la prudence et à la responsabilité individuelle et / ou collective pour ne pas dire d'affolement. D'où l'entrée en vigueur de slogans qui tendent à personnaliser la lutte, c'est-à-dire on appelle chacun à prendre ses propres dispositions pour briser la chaine de contagion de la maladie. La communication change de paradigme, on part de l'individuel au collectif.

Ce passage ou ciblage communicationnel s'exprime par des énoncés sloganisés tels que Takkal sa masque, Aar sa boppou Aar sa mbokkou (Porte ton masque! protège toi pour protéger ton prochain !) du mouvement Matal de Keur Mbaye Fall $\left[{ }^{10}\right]$. L'emploi de l'impératif injonctionnel de la deuxième personne du singulier wolof vise à effacer toute ambigüité communicationnelle: le destinataire est «tu», qui représente tout le monde mais de manière individuelle. Chacun se sent concerné et se considère comme étant même le principal destinataire du message. « Tu » porte sur ses épaules la responsabilité de protéger son vis-àvis. On lui impute la responsabilité de tout manque de sa part pouvant causer la contamination à son concitoyen. Au-delà de sa propre protection, on cherche à le rendre plus prudent, plus vigilant pour participer d'une manière ou d'une autre à la rupture de la série des contaminations du virus. Cette stratégie communicationnelle est très frappante et efficace car elle joue sur la psychologie de la population. L'atteinte de la maladie par manque de respect des mesures sanitaires à observer ou pas suscite même au sein de la société une sorte stigmatisation des voisins à fortiori la contaminer à son voisin, qui serait une responsabilité psychologique lourde à porter. L'énoncé «switché » (wolof-français) jëf ngir aar sa ma njaboot, pour vous protéger et protéger votre famille portez un masque lancé par les services de Orange info $\left[{ }^{11}\right]$ s'inscrit dans le sillage de responsabilisation de chacun. On adosse la charge de protection de la famille contre le virus à chaque membre de la famille. Ici, on joue sur la sensibilité et l'amour de chacun envers sa propre famille. On fait de la lutte une affaire de famille d'abord avant d'en faire une affaire de communauté au sens large. Portez un masque qui est l'élément moteur de l'énoncé est l'action attendue de chaque individu pour le bien de sa propre famille.

A la limite, l'on peut concevoir de tels énoncés ou slagans comme des stratégies communicationnelles qui s'assimilent à des mises en garde faites à l'endroit

\footnotetext{
${ }^{10}$ Une commune de Dakar

${ }^{11}$ Pris dans le site https://daancovid19.sn/masque/
}

de la population. Les slogans «mises en garde » s'apparentent à un affolement sous la forme d'injonction. Implicitement, ils renvoient à des risques ou dangers de mort liés à la gravité de la maladie. De tels énoncés sont de véritables «dissuadeurs», ils invitent les sénégalais à la vigilance, à la prudence dans ce contexte de pandémie.

Cette approche discursive sous l'apparence de la suggestion s'assimile à une alerte destinée aux populations dans le cadre de lutte contre le virus. Cette démarche communicative présuppose un danger que court la collectivité, et qui dépend de ses comportements attendus pour se sauver et sauver les autres. C'est un appel à la vigilance, à la prudence et au respect de toutes les mesures confondues prises pour endiguer l'expansion du virus au sein de la société. Les énoncés Takkal sa masque, Aar sa boppou Aar sa mbokkou (Porte ton masque ! protège toi pour protéger ton prochain !) et jëf ngir aar sa ma njaboot, pour vous protéger et protéger votre famille portez un masque comportent des actes de langage à valeur perlocutoire étant donné qu'ils délivrent des messages qui responsabilisent sans forcément le dire. Cette stratégie discursive à visée purement persuasive dans la communication de crise consiste à : « faire Prendre aux populations sénégalaises conscience de l'existence et de la gravité de la maladie ; faire Comprendre aux populations sénégalaises les manifestations de la maladie et les gestes barrières à observer pour s'en prémunir ; faire Adopter aux populations sénégalaises les comportements attendus pour se sauver et sauver les autres. » (Falilou Ba, 2020) $\left[^{12}\right]$. Les concepts et slogans employés ici pour la lutte contre le coronavirus constituent des techniques inclusives, suggestives, sensibilisatrices de communication pour les populations ciblées.

\section{CONCLUSION}

Dans cette analyse, nous avons constaté que la communication de crise sanitaire en général de la lutte contre la pandémie du COVID 19 se veut être une communication transparente, bien adaptée et évaluée suivant les étapes de l'évolution de la pandémie. Les slogans et concepts étudiés dans cet article mettent à nu d'une manière explicite ou implicite tous les risques sanitaires encourus par la population. Ils permettent également à faire prendre conscience à la population des conséquences mortelles de la propagation du virus. Cette communication à travers des concepts et slogans poignants et frappants sur la lutte contre la pandémie de la pandémie au Sénégal invite la cible (population) à s'impliquer activement dans le strict respect des mesures préventives. Les communicants adoptent un

\footnotetext{
${ }^{12}$ Pris dans le site : http//www.aps.sn/actualites/societe/sante/article/ Covid19-la-mairie-de-dakar-passe-a-l-offensive.
} 
Daouda NGOM., Sch Int J Linguist Lit, Mar, 2021; 4(3): 80-87

processus de sensibilisation à travers une démarche communicationnelle continue, dynamique, inclusive et bien ajustée. La communication constitue un outil efficace dans la gestion d'une crise sanitaire telle que la présente pandémie du COVID 19. Elle est levier incontournable dans l'exposition et la présentation des caractéristiques de la pandémie afin de mieux la gérer, la réduire ou l'éliminer définitivement.

\section{REFFERENCE}

1. ADAM, J. M., (1999). L'Argumentation publicitaire. Rhétorique de l'éloge et de la persuasion. Paris: Nathan Université.

2. BACHMANN, C., LINDENFELD, J., \& SIMONIN, J., (1981). Langage et communications sociales. Paris: Hatier-Crédit.

3. BOYER, H., (1996). Eléments de sociolinguistique: Langue, Communication et Société. Paris: DUNOD, $2^{\mathrm{em}}$ éd.

4. BRETON Philippe. (2000). La Parole manipulée. Paris: La Découverte.

5. CALVET, Louis-Jean. (1978). « Des mots sur les murs, le marquage linguistique du territoire » in Migrants Formation, $\mathrm{n}^{\circ} 83$, déc.

6. CHARAUDEAU, P., (2005) Le discours politique. Les masques du pouvoir. Paris: Vuibert.

7. CISSE, M., (2009). «Mouvements argumentatifs dans les textes politiques. Analyse sémiodiscursive de l'allocution de Nicolas Sarkozy à l'université Cheikh Anta Diop » in Annales de la FLSH, n³9/A, p : 25(44).

8. EDDINE K, J., et Cie. (2005). Crise sanitaire et stratégie de communication.

9. GIANNACCARO, F., (2021) « Communication en temps de crise : le poids des mots » in Risquesinfo, $\mathrm{n}^{\circ} 15$,

10. Hupet, M., \& Nespoulous, J.L., (1993). «La pragmatique » in Tendances actuelles en Linguistiques générale. Paris, Delachaux et Niestlé.

11. KENNED, Y., Georges. (1972).The art of persuasion in Greece. New Jersey: Princeton University Press.

12. LIBAERT, T.H., (2015). Communication de crise. Quatrième édition, DUNOD.

13. NGOM, D., (2017). «Langues nationales et communication politique au Sénégal, Essai typologique des stratégies de persuasion à travers quelques dénominations wolof données aux coalitions politiques des élections locales du 29 Juin 2014: le cas du département de Dakar » in Langues et Littératures, Revue du Groupe d'études linguistiques et littéraires, $\mathrm{n}^{\circ} 2$, Janvier; 45(65), UGB, Sénégal.

14. Reboul, O., (1975). Le slogan, Bruxelles, Complexe.

15. REY, A., Le Grand Robert de la langue française, dictionnaire français, deuxième édition dirigée par Alain Rey.

16. SEARLE, J.R., (1972). Les actes de langage. Paris: Hermann. 